Please do not remove this page

RMIT

UNIVERSITY

\title{
Weaving quantum optical frequency combs into continuous-variable hypercubic cluster states
}

Wang, Pei; Chen, Moran; Menicucci, Nicolas; Pfister, Olivier

https://researchrepository.rmit.edu.au/esploro/outputs/9921862566601341/filesAndLinks?institution=61RMIT_INST\&index=null

Wang, P., Chen, M., Menicucci, N., \& Pfister, O. (2014). Weaving quantum optical frequency combs into continuous-variable hypercubic cluster states. Physical Review A - Atomic, Molecular, and Optical Physics, 90(3), 1-8. https://doi.org/10.1103/PhysRevA.90.032325

Document Version: Accepted Manuscript

Published Version: https://doi.org/10.1103/PhysRevA.90.032325

Repository homepage: https://researchrepository.rmit.edu.au

(c) 2014 American Physical Society.

Downloaded On 2023/04/26 15:28:18 +1000 
Thank you for downloading this document from the RMIT Research Repository.

The RMIT Research Repository is an open access database showcasing the research outputs of RMIT University researchers.

RMIT Research Repository: http://researchbank.rmit.edu.au/

\section{Citation:}

Wang, P, Chen, M, Menicucci, N and Pfister, O 2014, 'Weaving quantum optical frequency combs into continuous-variable hypercubic cluster states', Physical Review A - Atomic, Molecular, and Optical Physics, vol. 90, no. 3, 032325, pp. 1-8.

See this record in the RMIT Research Repository at:

https://researchbank.rmit.edu.au/view/rmit:34275

Version: Accepted Manuscript

Copyright Statement:

(C) 2014 American Physical Society

\section{Link to Published Version:}

http://dx.doi.org/10.1103/PhysRevA.90.032325 


\title{
Weaving quantum optical frequency combs into continuous-variable hypercubic cluster states
}

\author{
Pei Wang, ${ }^{1}$ Moran Chen, ${ }^{1}$ Nicolas C. Menicucci, ${ }^{2}$, , and Olivier Pfister ${ }^{1,}{ }^{1}$ \\ ${ }^{1}$ Department of Physics, University of Virginia, Charlottesville, Virginia 22903, USA \\ ${ }^{2}$ School of Physics, The University of Sydney, Sydney, NSW 2006, Australia
}

(Dated: August 4, 2018)

\begin{abstract}
Cluster states with higher-dimensional lattices that cannot be physically embedded in three-dimensional space have important theoretical interest in quantum computation and quantum simulation of topologically ordered condensed-matter systems. We present a simple, scalable, top-down method of entangling the quantum optical frequency comb into hypercubic-lattice continuous-variable cluster states of a size of about $10^{4}$ quantum field modes, using existing technology. A hypercubic lattice of dimension $D$ (linear, square, cubic, hypercubic, etc.) requires but $D$ optical parametric oscillators with bichromatic pumps whose frequency splittings alone determine the lattice dimensionality and the number of copies of the state.
\end{abstract}

PACS numbers: 03.65.Ud, 03.67.Bg, 42.50.Dv, 03.67.Mn, 42.50.Ex, 42.65.Yj

\section{INTRODUCTION}

Quantum computing promises exponential speedup for particular computational tasks, such as integer factoring [1], which bears importance for encryption technology, and quantum simulation [2], which holds vast scientific potential. The two main flavors of quantum computing are the circuit model [3] and the measurement-based model [4] and, in particular, one-way quantum computing [5, 6], in which all entanglement resources are generic and provided up front in the form of a cluster state [7, 8] with square-lattice [5, 9] structure. One-way quantum computing is experimentally appealing because measurements are often easier to implement than coherent control of quantum information [10].

Nevertheless, scalable generation of cluster states remains a formidable challenge toward which many subfields of physics have converged [11]. Most proposed experimental implementations are "bottom-up" approaches, in which qubits are brought together and entangled one by one [11]. Alternatively, individual quantum modes of light, or "qumodes," can be entangled into continuous-variable cluster states [8] and used for universal one-way quantum computing [6, 9] based on continuous-variable (CV) quantum information [12[15]. Each qumode is an independent quantum oscillator mode of the electromagnetic field with amplitude- and phasequadrature field observables, $\hat{q}=\frac{1}{\sqrt{2}}\left(\hat{a}+\hat{a}^{\dagger}\right)$ and $\hat{p}=$ $\frac{i}{\sqrt{2}}\left(\hat{a}^{\dagger}-\hat{a}\right)$, the analogues of oscillator position and momentum. A temporal bottom-up approach has been used to sequentially generate the largest one-dimensional cluster state ever created to date [16]: 10,000 qumodes, only available two at a time - which still allows quantum computing [17, 18].

The only two "top-down" approaches, to our knowledge, are ultracold neutral atoms undergoing a Mott insulator transition in an optical lattice [19] and the novel method discovered by Menicucci, Flammia, and Pfister [20, 21] for generating vast square-grid cluster states over the qumodes of the

\footnotetext{
*ncmenicucci@gmail.com

$\dagger$ opfister@virginia.edu
}

quantum optical frequency comb (QOFC) of a single optical parametric oscillator (OPO). Qumode-based implementations promise massive scalability in resource-state generation but, for quantum computing, will require non-Gaussian processing [22] and a fault-tolerant encoding of qumodes due to errors from finite squeezing [9, 23] and photon loss [24]. Photon-number-resolving detection [25] is a crucial enabler in this respect. Moreover, it is important to note here that the existence of a fault-tolerance threshold for CV quantum computing has now been proved [26].

The proposal of Ref. [27] was realized in 2011, with a record 60 qumodes in the QOFC of a single OPO simultaneously and identically entangled into 15 copies of a quadripartite cluster state with a square graph [28]. More recently, scalable dual-rail quantum-wire cluster states were experimentally realized over the QOFC of a single OPO: one the one hand, one 60-qumode copy and, on the other other hand, two independent 30-qumode copies were fully characterized [29]. In this work like in the work of Ref. [28], all qumodes were simultaneously available and the number of involved qumodes was only restricted by a technical limitation: limited local oscillator tunability in the measurement technique. A recent characterization of the OPO gain bandwidth shows that at least 6,700 qumodes, in lieu of 60 , should actually be involved [30]. The experimental confirmation of this assertion is in progress.

In this article, we propose a natural extension of the aforementioned dual-rail quantum wire generation-which we review below-to generating $\mathrm{CV}$ cluster states with hypercubiclattice graphs. Moreover, generating large qumode squarelattice cluster states also allows one to simulate difficult measurements on topologically ordered systems of oscillators [31].

While a square (2-hypercube) lattice is sufficient for universal one-way quantum computation [5, 6], error thresholds two orders of magnitude higher than with concatenated encodings are achievable using qubit cluster states with cubic lattices [32]. This is based on the error-correction properties of Kitaev's surface code [33], which is closely related to both qubit [34] and qumode [35] cluster states.

Finally, hypercubic-lattice cluster states are likely to have a similar connection to four-dimensional surface-code 
states [36]. When these codes are implemented as the ground space of a local Hamiltonian, they have remarkable selfcorrection properties. Our optical construction methods circumvent the limitations of a three-dimensional world, enabling simulation of measurements on these systems and possibly paving the way for Hamiltonian-based implementations, for instance in circuit QED [37, 38].

Previous proposals for scalable construction of CV multipartite entangled states using a single OPO [20, 21, 27, 28, 39, 40] required mode-concurrent interactions within the OPO and no extraneous interactions. Using insight from temporalmode construction methods [16, 18] and using recent studies of errors in CV cluster-state generation [18, 41], we here relax the latter requirement and propose a novel, simple setup using $D$ OPOs, each with a two-frequency pump-in contrast to the complicated 15-frequency pump-spectrum OPO of Refs. [20, 21] - and the same free spectral range (FSR), as the source of a multitude of frequency-encoded two-modesqueezed (TMS) states [42], which are approximations of the Einstein-Podolsky-Rosen (EPR) states [43].

We first show that when qumodes are grouped by frequency into logical collections known as macronodes [20, 21], these become naturally arranged into linear $(D=1)$, square $(D=2)$, cubic $(D=3)$, and hypercubic $(D=4)$ lattices by appropriate choices of the two OPO pump frequencies. We then derive the corresponding final CV cluster state, obtained by action of an interferometer within all macronodes. As with temporal qumodes [18], a significant advantage of using frequency-encoded qumodes is that the same optical interferometer can act on all macronodes at once, enabling huge scaling in the size of the generated states with a constant number of optical elements. A crucial feature of the frequencyqumode encoding not present when using temporal qumodes is that all qumodes exist simultaneously, enabling measurements to be made in any order. Finally, we expound the experimental verification of the state by use of the established techniques of Ref. [28, 29].

Scalability comes in three different varieties in this work:

1. Scaling the size of the cluster state-i.e., the number of entangled qumodes in each OPO. With a FSR $\Delta \omega=$ $0.95 \mathrm{GHz}$ [28, 29] and a phasematching bandwidth $\Omega$ of at least 3.2 THz with a flat top, as measured in [30] in a 1-cm periodically poled (PP) $\mathrm{KTiOPO}_{4}$ (KTP) nonlinear crystal, we expect $\Omega / \Delta \omega \geqslant 6,700$ qumodes per OPO.

2. Scaling the dimensionality $D$ of the lattice representing the cluster state; $D$ is the number of OPOs.

3. Scaling the number of copies of the desired cluster state, determined by the frequency difference between the two pump fields of each OPO.

\section{TMS-STATE GENERATION}

Our experimental system is based on a polarizationdegenerate OPO [28, 29] containing two identical PPKTP
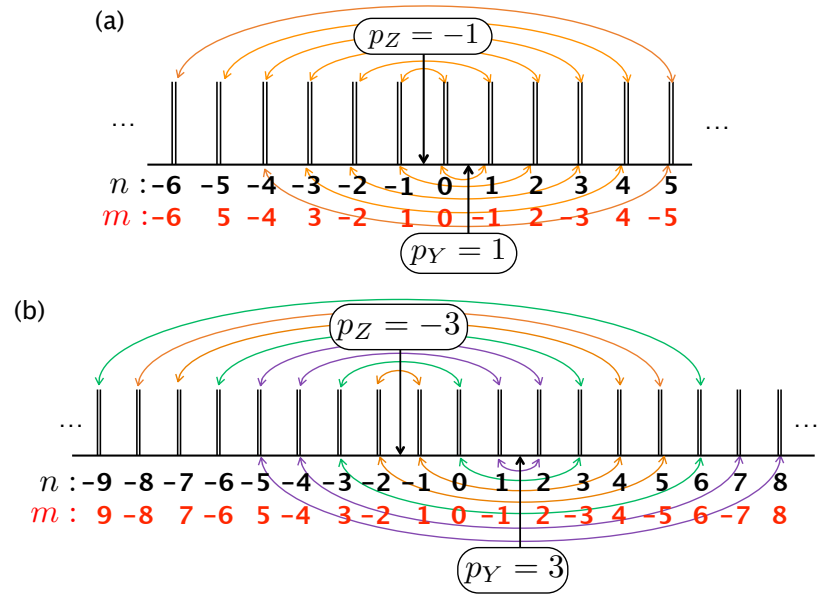

FIG. 1. The phasematched QOFC interactions in two different OPOs, with $Y$ - and $Z$-polarized pump indices $p_{Y}$ and $p_{Z}$ (black arrows). The qumodes are denoted by vertical lines (with orthogonal polarizations at the same frequency slightly separated for clarity) labeled by frequency index $n$ and node index $m$ (in red). The curved arrows denote the nonlinear interactions ( $Z Z Z$, top; $Y Y Y$, bottom), each of which becomes an edge of weight 1 in the OPO's H-graph [21, 44] and generates corresponding TMS states. (a) The QOFC of a single OPO with $p_{Y}=-p_{Z}=\Delta m=1$, which produces a single chain of interactions between adjacent node indices. (b) The QOFC of a single OPO with $p_{Y}=-p_{Z}=\Delta m=3$, which produces interactions between all pairs of node indices $m$ (red) separated by three units. This can also be interpreted as producing three independent chains (colored arrows) of the type obtained in (a).

crystals oriented at $90^{\circ}$ from each other, with the first (second) quasiphasematching the $Z Z Z(Y Y Y)$ interaction, as defined by the polarization directions of pump and downconverted fields, $Z(Y)$ being the horizontal (vertical) direction.

The QOFC created by the optical cavity is a collection of equally spaced, well-resolved qumodes at frequencies $\omega_{n}=$ $\omega_{0}+n \Delta \omega$, with $\omega_{0}$ an arbitrary offset, $n \in \mathbb{Z}$ an integer labeling the frequency index within the comb, and $\Delta \omega$ the FSR of the OPO cavity. Pump light at frequency $\omega_{\text {pump }}$ in the crystal will downconvert into photons of frequencies $\omega_{n_{1}}$ and $\omega_{n_{2}}$ such that

$$
\omega_{\text {pump }}=\omega_{n_{1}}+\omega_{n_{2}}=2 \omega_{0}+\Delta \omega\left(n_{1}+n_{2}\right) .
$$

We rewrite this phasematching condition by defining the pump index

$$
p:=\frac{\omega_{\text {pump }}-2 \omega_{0}}{\Delta \omega}=n_{1}+n_{2} .
$$

Nondegenerate downconversion, which creates TMS states with no single-mode squeezing, requires an odd pump index $p$ so that $n_{1} \neq n_{2}$. Without loss of generality, we assume that $n_{1}$ is odd and that $n_{2}$ is even from this point forward.

For convenience, we now replace each mode index with a macronode index

$$
m:=(-1)^{n} n
$$


The phasematching condition then becomes a difference condition on macronode indices:

$$
p=m_{2}-m_{1} .
$$

Since $m_{2}$ is assumed even and $m_{1}$ is assumed odd, we can repeatedly add 2 to both and still satisfy the condition. This relation therefore produces a two-step-translationally invariant set of interactions (for each polarization) with respect to the macronode indices [Figs. 1(a); 2(a); and 5(a), top].

We can write the Hamiltonian in the interaction picture with a single classical undepleted pump. The well-known TMS Hamiltonian is

$$
\hat{H}=i \hbar \kappa \hat{a}_{1}^{\dagger} \hat{a}_{2}^{\dagger}+\text { H.c. },
$$

where $\kappa>0$ is the overall nonlinear coupling strength. We can write this in terms of the adjacency matrix of a $\mathrm{H}$ (amiltonian)-graph [21, 27, 40, 44]

$$
\mathbf{G}=\left(\begin{array}{ll}
0 & 1 \\
1 & 0
\end{array}\right)=\stackrel{1}{\bullet}
$$

with components $G_{j k}$, as follows:

$$
\hat{H}[\mathbf{G}]=i \hbar \frac{\kappa}{2} \sum_{j k} G_{j k} \hat{a}_{j}^{\dagger} \hat{a}_{k}^{\dagger}+\text { H.c. }
$$

In this simple case, the two-mode interaction is purely nondegenerate (i.e., $\mathbf{G}$ is purely off-diagonal), and we have a graph with no self-loops. We will eschew degenerate interactions (self-loops in G) throughout this paper. We now introduce more elaborate H-graphs, which will be plugged into Eq. (7) to represent more complicated interactions.

\section{MACRONODE LATTICE SETUP}

We now show that the TMS states generated by $D$ OPOs are naturally arranged by the phasematching condition in a $D$-hypercubic lattice of frequency-degenerate macronodes. In section [V] we will describe the interferometer that acts within each macronode to generate cluster entanglement.

\section{A. Scaling the graph valence}

We consider a collection of $D$ OPOs, each of which pumped by two monochromatic fields of distinct frequencies and orthogonal polarizations, with OPO \# $j$ having pump in$\operatorname{dex} p_{j \varepsilon}$ per polarization $\varepsilon$. This implements the Hamiltonian

$$
\hat{H}=i \hbar \kappa \sum_{j=1}^{D} \sum_{\varepsilon \in\{Y, Z\}} \sum_{m_{j \varepsilon} \in 2 \mathbb{Z}+1} \hat{a}_{m_{j \varepsilon}}^{\dagger} \hat{a}_{m_{j \varepsilon}+p_{j \varepsilon}}^{\dagger}+\text { H.c. },
$$

which can be represented by $\hat{H}[\mathbf{G}]$ from Eq.(7) using the $\mathrm{H}$ graph

$$
\mathbf{G}=\bigoplus_{j=1}^{D} \bigoplus_{\varepsilon \in\{Y, Z\}} \bigoplus_{m_{j \varepsilon} \in 2 \mathbb{Z}+1}(\stackrel{1}{ })_{m_{j \varepsilon}, m_{j \varepsilon}+p_{j \varepsilon}} .
$$

To create the desired structures, we prescribe that

$$
p_{j Y}=-p_{j Z}=\Delta m_{j},
$$

which corresponds to an H-graph with exactly one edge between all pairs of macronodes separated by $\left|\Delta m_{j}\right|$, each of which produces a corresponding TMS state, as illustrated in Fig. 1

(1) Linear lattices: Figure 1(a) depicts the $\mathrm{H}$-graph of a single OPO (\#1) with $p_{1 Y}=-p_{1 Z}=\Delta m_{1}=1$ This graph is a collection of TMS state edges, which are shown reordered in Fig. 2(a), where all qumodes of same index define to a macronode and a linear structure is clearly visible. (We will see in section $[\mathrm{IV}$ that a Hadamard interferometer transforms this linear sequence of disconnected EPR edges into a dual-rail quantum entangled wire, or single quantum wire over macronodes, as was experimentally demonstrated in Ref. [29].)

Figure 1. b) shows an additional, remarkable feature of this construction: when $\left|\Delta m_{j}\right|>1$, the OPO will generate $\left|\Delta m_{j}\right|$ (here, 3) disjoint quantum wires. This generation of multiple quantum wires in a single OPO was also demonstrated in Ref. [29] and is the basis for generating higher-dimensional lattices, to which we now turn.

(2) Square lattice: We now imagine taking the quantumwire sequence of OPO \#1, as in Fig. 2(a), and "wrapping" it around a fictitious "cylinder," like a piece of thread around a spool [green wire in Fig. 2 b)]. We then employ a second OPO (\#2), with $p_{2 Y}=-p_{2 Z}=\Delta m_{2}=7$ here, to create 7 additional quantum-wire sequences [purple wires in Fig. [2(b)] whose macronodes exactly overlap with those of the first (spiraling) wire and bridge the spiral's coils with graph edges along the second lattice dimension (i.e., along the cylinder's axis), which will result in a square lattice with twisted cylindrical topology [Fig. 2(b)]. For a cylinder of circumference $M_{2}$ in units of macronode-index spacing, such a construction requires $\Delta m_{1}=1$ (for the wrapped wire) and $\Delta m_{2}=M_{2}$ (for the cross-links).

(3) Cubic lattice: This method can be extended to higherdimensional lattices by using a fractal procedure, treating the twisted cylindrical lattice from the previous step as the linear resource to itself be wrapped around another cylinder [Fig. 2(c)], with an additional OPO used to create edges along the axis of the new cylinder and between adjacent macronodes along the new cylinder axis. For example, by first wrapping the wire around a cylinder of circumference $M_{2}$ and then wrapping that entire structure around a second cylinder of circumference $M_{3}$, we can create all the required macronode links with 3 OPOs with $\Delta m_{1}=1, \Delta m_{2}=M_{2}$, and $\Delta m_{3}=M_{2} M_{3}$. This results in a cubic lattice in the macronodes with twisted toroidal topology in the first two dimensions and linear topology in the third.

(4) Hypercubic lattices: Continuing this fractal progression weaves hypercubic lattices from macronodes. In general, for a $D$-dimensional hypercubic lattice, one employs $D$ OPOs with $\Delta m_{j}=\prod_{k=1}^{j} M_{k}$ for OPO \# $j$ (and $M_{1}=1$ ). These lattices have twisted toroidal topology in the first $D-1$ dimensions and are linear in the $D^{\text {th }}$ one. 


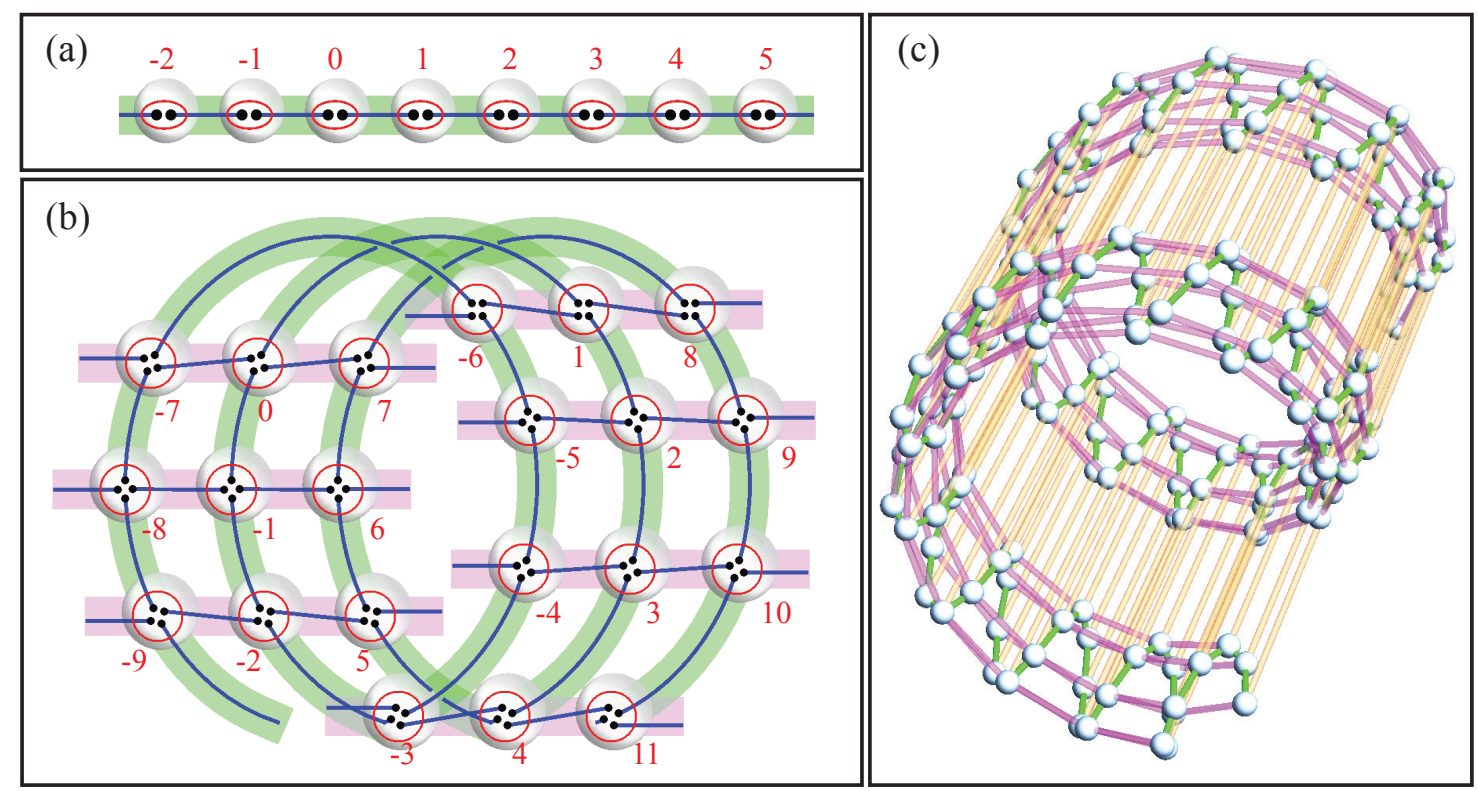

FIG. 2. Arrangements of the TMS states ${ }^{1}$ - qumodes into (a) linear, (b) square-lattice, and (c) cubic-lattice configurations (with $M_{2}=7$ and $M_{3}=13$ ) by grouping together frequency-degenerate qumodes into macronodes (red circles or white spheres) labeled by macronode indices (red numbers). See text for details. In (c), only the macronode connective structure is shown; individual qumodes and their connections are hidden for clarity. The macronode connections created by OPO \#1, \#2, \#3 are drawn in green, purple, yellow transparent tubes.

\section{B. Scaling the number of independent copies of the graph}

The same $D$ OPOs can create $M$ copies of a $D$-hypercubic lattice from (4) above, if OPO \# $j$ has pump indices

$$
p_{j(Z, Y)}= \pm M \Delta m_{j}+(M-1)
$$

and if we now label each macronode by a two-component compound macronode index $m^{(k)}$ for previous macronode index $m$ within lattice $k \in \mathbb{Z}_{M}$, then the frequency indices become

$$
\begin{array}{ll}
n=\left(M m^{(k)}+k\right), & \text { if } m^{(k)} \text { is even } \\
n=-\left(M m^{(k)}+k\right)+(M-1), & \text { if } m^{(k)} \text { is odd. }
\end{array}
$$

An example of making three copies of linear lattice cluster states is shown in Fig. 3 Following the dimension buildingup precedure from (2) to (4), multiple copies of square [Fig. 2(b)], cubic [Fig. 2(c)] and hypercubic lattice cluster states can be constructed.

\section{MACRONODE LATTICE ENTANGLEMENT}

The quantum-wire sequences being appropriately arranged in a $D$-hypercubic pattern, we first describe the entanglement step, which is to interfere all qumodes within each (frequencydegenerate) macronode [18] by use of a Hadamard interferometer. The formal justification and proof of this will employ the graphical calculus for Gaussian pure states [44, 45].

\section{A. Experimental construction of hypercubic lattice clusters}

In the Heisenberg picture, the action of an interferometer on $2 D$ qumodes ( $D$ frequencies, two polarizations) is modeled by the action of a unitary matrix $\mathbf{U}$ on a vector of qumode annihilation operators $\hat{\mathbf{a}}=\left(\hat{a}_{1}, \ldots, \hat{a}_{2 D}\right)^{\mathrm{T}}$. Here, we need the interferometer to be balanced, i.e., all entries of $\mathbf{U}$ to have equal magnitude.

When $2 D$ is a multiple of 4 , up to 668 and possibly higher [46], $\mathbf{U}$ can be chosen to be a $2 D \times 2 D$ Hadamard matrix $\mathbf{R}$. We restrict ourselves to this case for simplicity, leaving the general case to future work. For $D=1$, a $\frac{\pi}{8}$ half-wave plate (HWP) acts as a balanced beamsplitter on polarization modes with $\mathbf{R}$, in this case, being

$$
\mathbf{H}_{1}:=\frac{1}{\sqrt{2}}\left(\begin{array}{cc}
1 & 1 \\
1 & -1
\end{array}\right) \text {. }
$$

Using the Sylvester construction of Hadamard matrices [47], we can obtain the balanced $2 D$-splitter matrix

$$
\mathbf{H}_{D}:=\mathbf{H}_{1}^{\otimes D}
$$

which can be implemented using balanced beamsplitters [48, 49] or, equivalently, using $\frac{\pi}{8}$ HWPs and polarizing beamsplitters (PBSs). Figure 4 shows the experimental setup to generate cluster states with linear, square-lattice, and 4-hypercubiclattice graphs. Each compact setup builds on the previous one, akin to the fractal construction of Fig. 2. All ring OPO cavities must be of identical FSR and held to the same exact resonant frequency, e.g., by Pound-Drever-Hall servo locks to the same counterpropagating reference laser beam [28, 29]. 


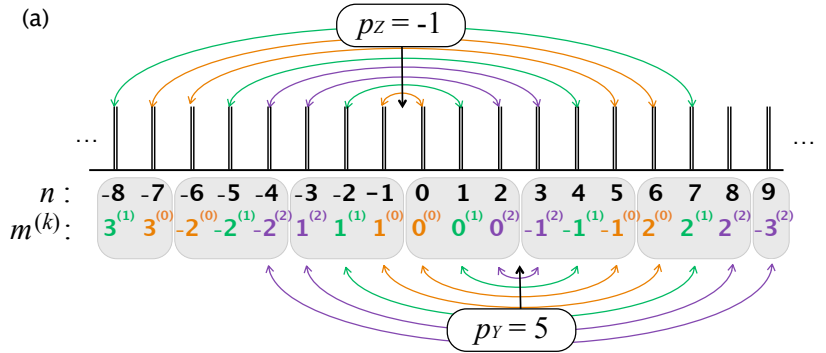

(b)
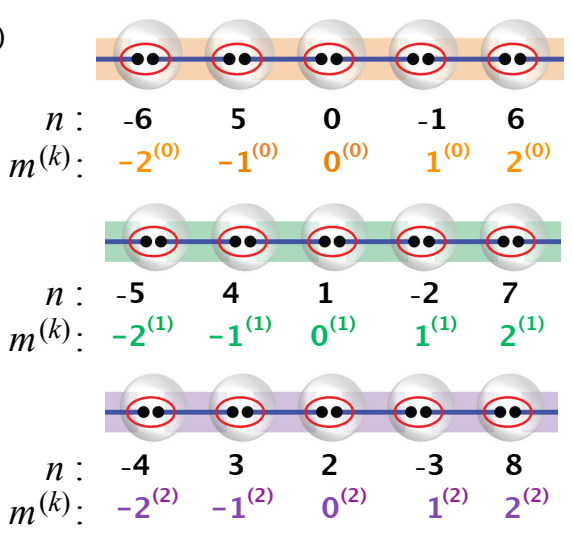

FIG. 3. An example of making three copies of linear lattice cluster states. Different colors indicates different linear lattice cluster states. (a) The compound macronode index $m^{(k)}$ is used instead of the macronode index $m$. In this case, $\Delta m_{j}=1, M=3, k \in\{0,1,2\}$. (b) The TMS states can be arranged into three groups, each group will independently form a linear lattice cluster state. Starting from this, by applying the procedure from (2)-(4) multiple copies of lattice cluster states with higher dimension can be constructed.

\section{B. Theoretical construction of hypercubic lattice clusters}

Any $N$-mode Gaussian pure state has a position-space wavefunction of the form [44]

$$
\psi_{\mathbf{Z}}(\mathbf{q})=\operatorname{det}\left(\frac{\operatorname{Im} \mathbf{Z}}{\pi}\right)^{\frac{1}{4}} \exp \left(\frac{i}{2} \mathbf{q}^{\mathrm{T}} \mathbf{Z} \mathbf{q}\right)
$$

up to displacements, for some complex, symmetric matrix $\mathbf{Z}$ with $\operatorname{Im} \mathbf{Z}>0$. $\mathbf{Z}$ can be interpreted as the adjacency matrix of an $N$-node, undirected, complex-weighted graph and evolves under Gaussian unitary operations (in the Schrödinger picture) according to simple graph transformation rules [44]: Starting with the $D$ OPOs represented by $\mathbf{G}$ from Eq. (9), when the Hamiltonian $\hat{H}(\mathbf{G})$ in Eq. (8) is applied on the vacuum state for time $t$, the output state is a Gaussian pure state with graph

$$
\mathbf{Z}_{0}=i \exp (-2 \alpha \mathbf{G})
$$

where $\alpha=2 \kappa t>0$ is an overall squeezing parameter. Crucially, since $\mathbf{G}$ is self-inverse [41, 44], this relation simplifies to

$$
\mathbf{Z}_{0}=i c \mathbf{I}-i s \mathbf{G}
$$

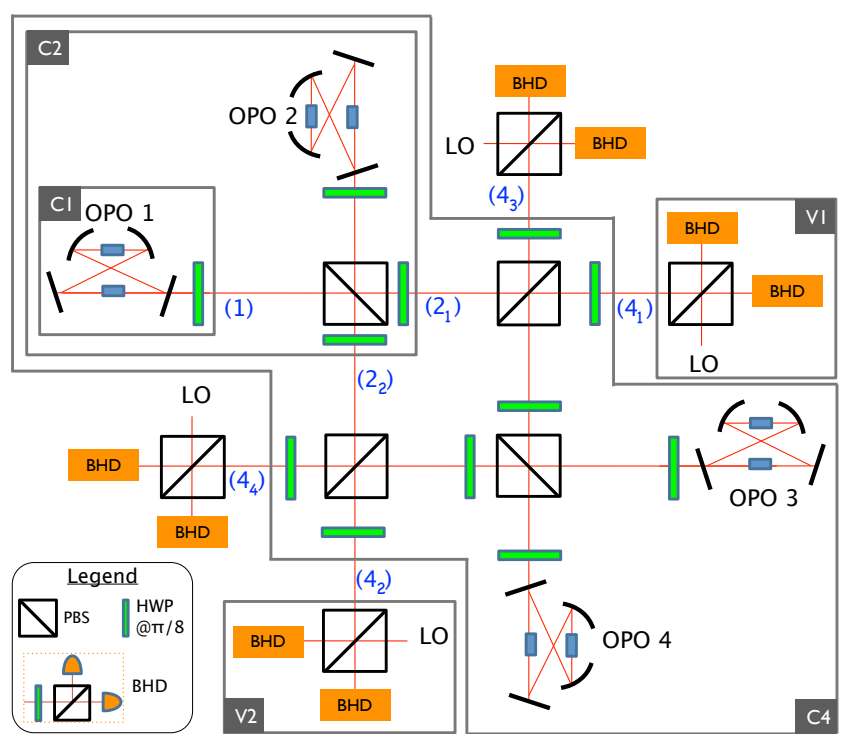

FIG. 4. Compact experimental setups for generating and verifying QOFC-based CV cluster states with linear, square-lattice, and 4hypercubic-lattice graphs. All polarizing beamsplitters (PBSs) transmit $Z$ and reflect $Y$, and all half-wave plates (HWPs) are at $\frac{\pi}{8}$ to the PBSs' axes. Box C1 generates at (1) a CV cluster state with linear topology as in Fig. 22a) and graph structure as shown in Fig. 5.a), which can be verified using two-tone balanced homodyne detection (BHD) in Box V1 (and omitting all the other optical elements). This was demonstrated experimentally in Ref. [29]. Box C2 builds on this setup to generate at $\left(2_{1,2}\right)$ a square-lattice $\mathrm{CV}$ cluster state with twisted cylindrical topology as in Fig. 2.b) and graph structure as shown in Fig. 5. b). This can be verified using Boxes V1 and V2. Box $\mathrm{C} 4$ further builds on this, generating at $\left(4_{1-4}\right)$ a 4-hypercubiclattice CV cluster state with toroidal topology in the first three dimensions and linear topology in the fourth, which can be verified using all BHD's. The BHDs contain a two-tone local oscillator (LO), phaselocked to the OPO and polarized at $\frac{\pi}{4}$ to the PBS's axis [28, 29].

where $c=\cosh 2 \alpha$, and $s=\sinh 2 \alpha$, resulting in a TMS state for each edge in $\mathbf{G}$ [Figs. 5(a), top, and 5(b), left].

We write the total interferometer as $\mathbf{R}=\bigoplus_{m \in \mathbb{Z}}\left(\mathbf{H}_{D}\right)_{m}$, which acts with $\mathbf{H}_{D}$ simultaneously on each macronode, evolving the state as [44]

$$
\mathbf{Z}_{0} \stackrel{\mathbf{R}}{\longmapsto} \mathbf{Z}=i c \mathbf{I}-i s \mathbf{R} \mathbf{G R}^{\mathrm{T}} .
$$

Since $\mathbf{R} \mathbf{G R}^{\mathrm{T}}$ is self-inverse, $\mathbf{Z}$ is equivalent [18]-up to trivial local phase shifts - to the approximate CV cluster state

$$
\mathbf{Z}_{C}=i \epsilon \mathbf{I}+t \mathbf{R} \mathbf{G} \mathbf{R}^{\mathrm{T}}
$$

where $\epsilon=\operatorname{sech} 2 \alpha$, and $t=\tanh 2 \alpha$. We focus on $\mathbf{Z}$ rather than $\mathbf{Z}_{C}$ for experimental simplicity but still refer to the former as a "CV cluster state" because the phase shifts can be absorbed entirely into mode-wise quadrature redefinitions [18].

As shown in Fig. 5, we can see after interfering the $2 D$ output qumodes of the OPOs by the balanced $2 D$-splitter, all qumodes within each macronode are entangled with all qumodes in the neighbor macronodes, thus creating a $D$ dimensional lattice cluster state. 

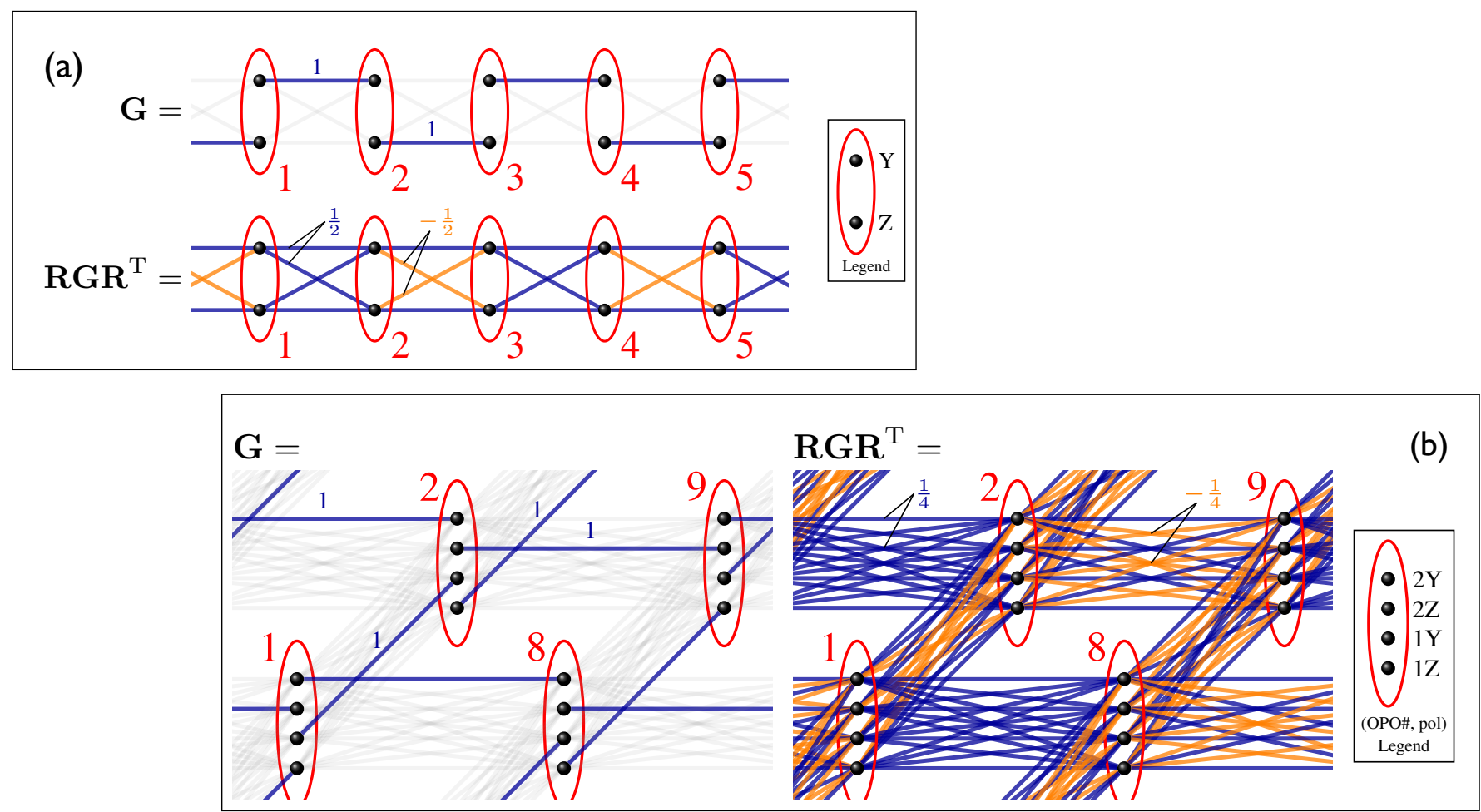

FIG. 5. Effect of the interferometers acting on the macronodes. In both (a) and (b), the combined H-graph G for the output of the OPO(s) is shown first, where the red circles indicate frequency-degenerate macronodes labeled by the red macronode indices (see text), with polarization [and OPO\# in (b)] as indicated in the legend. The state produced at the output of the OPOs has a graph [44] given by $\mathbf{Z}_{0}=i c \mathbf{I}-i s \mathbf{G}$ (see text), which corresponds to a collection of separable TMS states in accord with G. After the interferometer is applied (represented by the orthogonal matrix $\mathbf{R}$ ), a state with graph $\mathbf{Z}=i c \mathbf{I}-i s \mathbf{R} \mathbf{G} \mathbf{R}^{\mathrm{T}}$ results, which is phase-shift equivalent to the $\mathrm{CV}$ cluster state $\mathbf{Z}_{C}=i \epsilon \mathbf{I}+t \mathbf{R G} \mathbf{R}^{\mathrm{T}}$ (see text). The product $\mathbf{R} \mathbf{G R}^{\mathrm{T}}$, interpreted as an adjacency matrix, is visualized as the second graph and provides an intuitive picture for the resulting state, as well as its precise definition through $\mathbf{Z}$ or $\mathbf{Z}_{C}$ [44]. (a) An H-graph that is linear with respect to macronodes, a.k.a. dual-rail quantum wire [29], can be created from a single OPO with $\Delta m=1$ [Fig. 2[a)], and $\mathbf{R}$ represents the action of a balanced two-mode interferometer acting on each macronode. (b) An H-graph with a square-lattice graph on macronodes can be created from two OPOs with $\Delta m=1$ and $\Delta m=M_{2}$ [Fig. 2[b)]. Here, $M_{2}=7$, and $\mathbf{R}$ represents the action of a balanced four-mode interferometer acting on each macronode.

\section{STATE VERIFICATION}

A nullifier for a given state is any operator whose kernel contains the state. Like stabilizers [3, 50], nullifiers can be used to compactly represent states and track their evolution [44]. Any zero-mean Gaussian pure state $\left|\psi_{\mathbf{Z}}\right\rangle$ with graph $\mathbf{Z}$ satisfies a complete set of Schrödinger-picture nullifier relations [44]

$$
(\hat{\mathbf{p}}-\mathbf{Z} \hat{\mathbf{q}})\left|\psi_{\mathbf{Z}}\right\rangle=\mathbf{0}
$$

Note that linear combinations of nullifiers are still nullifiers. Also,

$$
\mathbf{Z}^{-1}=-i c \mathbf{I}-i s \mathbf{R} \mathbf{G} \mathbf{R}^{\mathrm{T}}
$$

and we can left-multiply Eq. 21, by $i \epsilon \mathbf{R}^{\mathrm{T}}$ and by $i \epsilon \mathbf{R}^{\mathrm{T}} \mathbf{Z}^{-1}$ to obtain, respectively,

$$
\begin{aligned}
{\left[i \epsilon \hat{\mathbf{p}}^{\prime}+\left(\hat{\mathbf{q}}^{\prime}-t \mathbf{G} \hat{\mathbf{q}}^{\prime}\right)\right]\left|\psi_{\mathbf{Z}}\right\rangle } & =\mathbf{0} \\
{\left[-i \epsilon \hat{\mathbf{q}}^{\prime}+\left(\hat{\mathbf{p}}^{\prime}+t \mathbf{G} \hat{\mathbf{p}}^{\prime}\right)\right]\left|\psi_{\mathbf{Z}}\right\rangle } & =\mathbf{0} .
\end{aligned}
$$

where

$$
\begin{aligned}
\hat{\mathbf{q}}^{\prime} & :=\mathbf{R}^{\mathrm{T}} \hat{\mathbf{q}} \\
\hat{\mathbf{p}}^{\prime} & :=\mathbf{R}^{\mathrm{T}} \hat{\mathbf{p}} .
\end{aligned}
$$

By taking linear combinations of Eqs. 23] and 24) and defining

$$
\begin{aligned}
& \hat{\mathbf{q}}_{\theta}^{\prime}:=\hat{\mathbf{q}}^{\prime} \cos \theta+\hat{\mathbf{p}}^{\prime} \sin \theta \\
& \hat{\mathbf{p}}_{\theta}^{\prime}:=\hat{\mathbf{q}}_{\theta+\pi / 2}^{\prime},
\end{aligned}
$$

we can generalize these to a continuum of $\theta$-indexed nullifier relations:

$$
\left[i \epsilon \hat{\mathbf{p}}_{\theta}^{\prime}+\left(\hat{\mathbf{q}}_{\theta}^{\prime}-t \mathbf{G} \hat{\mathbf{q}}_{-\theta}^{\prime}\right)\right]\left|\psi_{\mathbf{z}}\right\rangle=\mathbf{0}, \quad \forall \theta \in[0,2 \pi) .
$$

In particular, $\theta=0$ and $\theta=\frac{\pi}{2}$ yield Eqs. 233 and 24, respectively.

We consider the vector in parentheses in Eq. 29]:

$$
\mathbf{R}^{\mathrm{T}} \hat{\mathbf{q}}_{\theta}-t \mathbf{G R}^{\mathrm{T}} \hat{\mathbf{q}}_{-\theta}=: \hat{\mathbf{n}}_{\theta},
$$


which is comprised of simultaneously commuting observables known as approximate nullifiers [44] or variance-based entanglement witnesses [51]. Since $\mathbf{R}$ acts locally on frequencydegenerate qumodes and since $\mathbf{G}$ links each node to exactly one other of a different frequency, each component of $\hat{\mathbf{n}}_{\theta}$ contains exactly two frequencies and can be measured by the twotone balanced homodyne detection methods of Refs. [28, 29]. The theoretical covariance matrix [44] of $\hat{\mathbf{n}}_{\theta}$ is given by

$$
\operatorname{cov}\left(\hat{\mathbf{n}}_{\theta}\right)=\frac{\epsilon}{2}(\mathbf{I}-t \mathbf{G} \cos 2 \theta)
$$

vanishing in the large-squeezing limit $\alpha \rightarrow \infty$. Each element of $\hat{\mathbf{n}}_{\theta}$ therefore has a theoretical variance of $\epsilon$ (i.e., $\operatorname{sech} 2 \alpha$ ) units of vacuum noise.

Further application of the massively entangled QOFC to quantum information processing will require separating the frequencies. We are investigating the use of quantumoptics grade arrayed waveguide gratings [52] and of virtuallyimaged phase arrays [53], which have been successfully implemented in classical optical frequency combs [54].

\section{CONCLUSION}

We have proposed novel hypercubic-lattice cluster states, highly scalable in size, graph valence, and number of copies of the state, and we have detailed their experimental generation and characterization with remarkably compact and proven technology [28, 29]. The macronode-based implementation presented here and elesewhere [18] occurs naturally in quantum optics [16] and is becoming known to be a more efficient use of such cluster states for one-way quantum computing [55]. This work further motivates the development of a unified theoretical approach to macronodebased cluster states. Finally, the availability of large-scale, high-dimensional lattices invites theoretical and experimental investigations into the topological properties of these structures [31], including their high-dimensional incarnations [36].

\section{ACKNOWLEDGEMENTS}

We thank Matthew Broome, Robert Fickler, and Steven Flammia for discussions. This work was supported by the U.S. National Science Foundation grants No. PHY-1206029, No. PHY-0960047, and No. PHY-0855632. N.C.M. was supported by the Australian Research Council under grant No. DE120102204.
[1] P. W. Shor, in Proceedings, $35^{\text {th }}$ Annual Symposium on Foundations of Computer Science, edited by S. Goldwasser (IEEE Press, Los Alamitos, CA, Santa Fe, NM, 1994) pp. 124-134.

[2] R. P. Feynman, Int. J. Theor. Phys. 21, 467 (1982).

[3] M. A. Nielsen and I. L. Chuang, Quantum computation and quantum information (Cambridge University Press, Cambridge, U.K., 2000).

[4] D. Gottesman and I. L. Chuang, Nature (London) 402, 390 (1999).

[5] R. Raussendorf and H. J. Briegel, Phys. Rev. Lett. 86, 5188 (2001).

[6] N. C. Menicucci, P. van Loock, M. Gu, C. Weedbrook, T. C. Ralph, and M. A. Nielsen, Phys. Rev. Lett. 97, 110501 (2006)

[7] H. J. Briegel and R. Raussendorf, Phys. Rev. Lett. 86, 910 (2001).

[8] J. Zhang and S. L. Braunstein, Phys. Rev. A 73, 032318 (2006).

[9] M. Gu, C. Weedbrook, N. C. Menicucci, T. C. Ralph, and P. van Loock, Phys. Rev. A 79, 062318 (2009).

[10] H. J. Briegel, D. E. Browne, W. Dur, R. Raussendorf, and M. Van den Nest, Nat. Phys. 5, 19 (2009)

[11] T. D. Ladd, F. Jelezko, R. Laflamme, Y. Nakamura, C. Monroe, and J. L. O'Brien, Nature (London) 464, 45 (2010).

[12] S. Lloyd and S. L. Braunstein, Phys. Rev. Lett. 82, 1784 (1999).

[13] S. D. Bartlett, B. C. Sanders, S. L. Braunstein, and K. Nemoto, Phys. Rev. Lett. 88, 097904 (2002).

[14] S. L. Braunstein and P. van Loock, Rev. Mod. Phys. 77, 513 (2005)

[15] C. Weedbrook, S. Pirandola, R. García-Patrón, N. J. Cerf, T. C. Ralph, J. H. Shapiro, and S. Lloyd, Rev. Mod. Phys. 84, 621 (2012)
[16] S. Yokoyama, R. Ukai, S. C. Armstrong, C. Sornphiphatphong, T. Kaji, S. Suzuki, J. Yoshikawa, H. Yonezawa, N. C. Menicucci, and A. Furusawa, Nat. Photon. 7, 982 (2013).

[17] N. C. Menicucci, X. Ma, and T. C. Ralph, Phys. Rev. Lett. 104, 250503 (2010)

[18] N. C. Menicucci, Phys. Rev. A 83, 062314 (2011)

[19] M. Greiner, O. Mandel, T. Esslinger, T. W. Hänsch, and I. Bloch, Nature (London) 415, 39 (2002).

[20] N. C. Menicucci, S. T. Flammia, and O. Pfister, Phys. Rev. Lett. 101, 130501 (2008)

[21] S. T. Flammia, N. C. Menicucci, and O. Pfister, J. Phys. B, 42, 114009 (2009).

[22] J. Niset, J. Fiurášek, and N. J. Cerf, Phys. Rev. Lett. 102, 120501 (2009).

[23] M. Ohliger, K. Kieling, and J. Eisert, Phys. Rev. A 82, 042336 (2010).

[24] H. Cable and D. E. Browne, New J. Phys. 12, 113046 (2010)

[25] A. E. Lita, A. J. Miller, and S. W. Nam, Opt. Expr. 16, 3032 (2008).

[26] N. C. Menicucci, Phys. Rev. Lett. 112, 120504 (2014)

[27] H. Zaidi, N. C. Menicucci, S. T. Flammia, R. Bloomer, M. Pysher, and O. Pfister, Laser Phys. 18, 659 (2008), revised version at http://arxiv.org/pdf/0710.4980v3.

[28] M. Pysher, Y. Miwa, R. Shahrokhshahi, R. Bloomer, and O. Pfister, Phys. Rev. Lett. 107, 030505 (2011)

[29] M. Chen, N. C. Menicucci, and O. Pfister, Phys. Rev. Lett. 112, 120505 (2014)

[30] P. Wang, W. Fan, and O. Pfister, arXiv:1403.6631 [physics.optics] (2014). 
[31] T. F. Demarie, T. Linjordet, N. C. Menicucci, and G. K. Brennen, to appear in New Journal of Physics, arXiv:1305.0409 [quant-ph] (2013).

[32] R. Raussendorf, J. Harrington, and K. Goyal, Ann. Phys. (NY) 321, 2242 (2006).

[33] A. Kitaev, Ann. Phys. 303, 2 (2003), arXiv:quant-ph/9707021.

[34] Y. J. Han, R. Raussendorf, and L. M. Duan, Phys. Rev. Lett. 98, 150404 (2007)

[35] J. Zhang, C. Xie, K. Peng, and P. van Loock, Phys. Rev. A 78, $052121(2008)$

[36] E. Dennis, A. Kitaev, A. Landahl, and J. Preskill, J. Math. Phys. 43, 4452 (2001).

[37] R. J. Schoelkopf and S. M. Girvin, Nature (London) 451, 664 (2008)

[38] L. Aolita, A. J. Roncaglia, A. Ferraro, and A. Acín, Phys. Rev. Lett. 106, 090501 (2011)

[39] O. Pfister, S. Feng, G. Jennings, R. Pooser, and D. Xie, Phys. Rev. A 70, 020302 (2004).

[40] N. C. Menicucci, S. T. Flammia, H. Zaidi, and O. Pfister, Phys. Rev. A 76, 010302(R) (2007).

[41] N. C. Menicucci, in preparation (2013).

[42] Z. Y. Ou, S. F. Pereira, H. J. Kimble, and K. C. Peng, Phys. Rev. Lett. 68, 3663 (1992).
[43] A. Einstein, B. Podolsky, and N. Rosen, Phys. Rev. 47, 777 (1935).

[44] N. C. Menicucci, S. T. Flammia, and P. van Loock, Phys. Rev. A 83, 042335 (2011).

[45] R. Simon, E. C. G. Sudarshan, and N. Mukunda, Phys. Rev. A 37, $3028(1988)$

[46] D. Ż. Doković, Combinatorica 28, 487 (2008).

[47] T. K. Moon, Error correction coding (Wiley, 2005).

[48] M. Zukowski, A. Zeilinger, and M. A. Horne, Phys. Rev. A 55, 2564 (1997)

[49] Y. Ben-Aryeh, Opt. Comm. 283, 2863 (2010).

[50] D. Gottesman, Stabilizer codes and quantum error correction, Ph.D. thesis, California Institute of Technology, Pasadena, CA (1997), quant-ph/9705052

[51] P. Hyllus and J. Eisert, New J. Phys. 8, 51 (2006)

[52] D. Dai, Z. Wang, J. F. Bauters, M.-C. Tien, M. J. R. Heck, D. J. Blumenthal, and J. E. Bowers, Opt. Expr. 19, 14130 (2011)

[53] M. Shirasaki, Opt. Lett. 21, 366 (1996).

[54] S. A. Diddams, L. Hollberg, and V. Mbele, Nature (London) 445, 627 (2007).

[55] R. N. Alexander, S. C. Armstrong, R. Ukai, and N. C. Menicucci, arXiv:1311.3538 [quant-ph] (2013). 